\title{
MULTIPLE PERIODIC SOLUTIONS AND COMPLEX DYNAMICS FOR SECOND ORDER ODES VIA LINKED TWIST MAPS
}

\author{
ANNA PASCOLETTI, MARINA PIREDDU, FABIO ZANOLIN
}

\begin{abstract}
We consider some nonlinear second order scalar ODEs of the form $x^{\prime \prime}+f(t, x)=0$, where $f$ is periodic in the $t$-variable and show the existence of infinitely many periodic solutions as well as the presence of complex dynamics, even in the case of certain apparently "simple" equations. We employ a topological approach based on the study of linked twist maps (and suitable modifications of their geometry).
\end{abstract}

2000 AMS subject classification : 34C25, 37C25, 37D45.

keywords and phrases : Nonlinear second order ODEs, periodic solutions, chaotic dynamics, linked twist mappings.

\section{INTRODUCTION}

The principal aim of this paper is that of proving in a rigorous way (and without the need of computer assistance) the existence of infinitely many periodic solutions and the presence of chaotic dynamics for some nonlinear second order scalar ordinary differential equations (from now on ODEs) of the form

$$
x^{\prime \prime}+f(t, x)=0,
$$

where $f$ is periodic in the $t$-variable. In particular, we'll focus our study on some special cases of equation (1.1), like

$$
x^{\prime \prime}+g(x)=p(t) \text {, }
$$

or

$$
x^{\prime \prime}+q(t) g(x)=0,
$$

but we stress that our approach is, in principle, applicable also to more general equations, including examples in which a term depending on $x^{\prime}$ is present in (1.1).

THIS PAPER IS IN FINAL FORM AND NO VERSION OF IT IS SUBMITTED FOR PUBLICATION ELSEWHERE.

EJQTDE, Proc. 8th Coll. QTDE, 2008 No. 14, p. 1 
The tools which are employed are a combination of a careful but elementary phase-plane analysis with recent results on topological horseshoes and fixed points for maps defined on domains homeomorphic to the unit square of $\mathbb{R}^{2}[33,34,35]$.

Investigating our ODE models $[32,34,38]$ we have found that the Poincaré map associated to various first order planar systems presents some features which are typical of those usually connected with the so-called "linked twist maps". Thus a second goal of this work is that of providing a general geometrical setting which is particularly suited to deal with such kind of examples.

The plan of the article is the following. In Section 2 we explain the main topological theory, indicating also the precise definition of chaos we use. In Section 3 we introduce the framework of linked twist maps, concentrating in particular on their geometrical configurations and showing the connection with the theory previously exposed. These topological results are then applied to concrete examples in Section 4, where we show how the phase-portraits of some planar ODE systems recall the geometry associated to the linked twist maps. Part of the results collected in this work surveys some recent theorems from [34, $35,36,38,40]$; however, they are here presented from a different point of view, including also new applications.

\section{FiXED POINTS, PERIODIC POINTS AND CHAOTIC-LIKE DYNAMICS FOR A CLASS OF PLANAR MAPS}

In this section we introduce our main theoretical tools for the search of periodic points and complex dynamics of some continuous maps defined on two-dimensional domains. The results we are going to present may be considered as part of the theory of "topological horseshoes". Roughly speaking, with such a term we denote all those contributions in the broad area of chaotic dynamics where one proves the presence of some kind of complex behaviour typically associated to the celebrated Smale horseshoe (like the existence of a semiconjugation to the Bernoully shift on a set of $m$ symbols) by assuming relaxed assumptions on the maps under consideration, possibly replacing the usual hyperbolicity hypotheses with topological conditions which may require the knowledge of the behavior of a map only on some subsets of its domain (for instance, at the boundary of certain sets). Results in this direction were obtained by Easton [11], Burns and Weiss [5], Mischaikow and Mrozek [27], Szymczak [51], Zgliczyński [56, 57], Srzednicki and EJQTDE, Proc. 8th Coll. QTDE, 2008 No. 14, p. 2 
Wójcik [49], Kennedy and Yorke [15, 16, 17] and further developed in subsequent papers (see also [13, 14, 29, 39, 48, 58, 59, 60], just to quote some recent works in this area).

We start with a few definitions.

Let $X$ be a metric space. By a path $\gamma$ in $X$ we mean a continuous mapping $\gamma: \mathbb{R} \supseteq[a, b] \rightarrow X$. We also set $\bar{\gamma}:=\gamma([a, b])$. Of course, there is no loss of generality in assuming, as a basic interval, $[a, b]=[0,1]$. A sub-path $\sigma$ of $\gamma$ is the restriction of $\gamma$ to a compact subinterval of its domain. An arc is the homeomorphic image of the compact interval $[0,1]$, while an open arc is an arc without its end-points. We define a generalized rectangle as a set $\mathcal{R} \subseteq X$ which is homeomorphic to the unit square $\mathcal{Q}:=[0,1]^{2} \subseteq \mathbb{R}^{2}$. If $\mathcal{R}$ is a generalized rectangle and $h: \mathcal{Q} \rightarrow h(\mathcal{Q})=\mathcal{R}$ is a homeomorphism defining $\mathcal{R}$, we call contour $\vartheta \mathcal{R}$ of $\mathcal{R}$ the set

$$
\vartheta \mathcal{R}:=h(\partial \mathcal{Q})
$$

where $\partial \mathcal{Q}$ is the usual boundary of the unit square. Note that the contour $\vartheta \mathcal{R}$ is well defined and it is independent of the choice of the homeomorphism $h$. In fact, $\vartheta \mathcal{R}$ is also a homeomorphic image of $S^{1}$, that is, a Jordan curve (the image of a simple closed curve).

By an oriented rectangle we mean a pair

$$
\widetilde{\mathcal{R}}:=\left(\mathcal{R}, \mathcal{R}^{-}\right),
$$

where $\mathcal{R} \subseteq X$ is a generalized rectangle and

$$
\mathcal{R}^{-}:=\mathcal{R}_{l}^{-} \cup \mathcal{R}_{r}^{-}
$$

is the union of two disjoint $\operatorname{arcs} \mathcal{R}_{l}^{-}, \mathcal{R}_{r}^{-} \subseteq \vartheta \mathcal{R}$, called the left and the right components of $\mathcal{R}^{-}$. Since $\vartheta \mathcal{R}$ is a Jordan curve we have that $\vartheta \mathcal{R} \backslash\left(\mathcal{R}_{l}^{-} \cup \mathcal{R}_{r}^{-}\right)$consists of two open arcs. We denote by $\mathcal{R}^{+}$the closure of such open arcs, called $\mathcal{R}_{d}^{+}$and $\mathcal{R}_{u}^{+}$(the down and up components of $\mathcal{R}^{+}$). It is important to notice that we can always label the $\operatorname{arcs} \mathcal{R}_{l}^{-}$, $\mathcal{R}_{d}^{+}, \mathcal{R}_{r}^{-}$and $\mathcal{R}_{u}^{+}$, following the cyclic order $l-d-r-u-l$, and take a homeomorphism $h: \mathcal{Q} \rightarrow h(\mathcal{Q})=\mathcal{R}$ so that the left and right sides $(\{0\} \times[0,1]$ and $\{1\} \times[0,1])$ of $\mathcal{Q}$ are mapped onto $\mathcal{R}_{l}^{-}$and $\mathcal{R}_{r}^{-}$, while the lower and upper sides $([0,1] \times\{0\}$ and $[0,1] \times\{1\})$ of $\mathcal{Q}$ are mapped onto $\mathcal{R}_{d}^{+}$and $\mathcal{R}_{u}^{+}$, respectively (see Figure 1 ).

The following lemma is a classical result from plane topology. It plays a crucial role in our fixed point theorem (see Theorem 2.1 below) EJQTDE, Proc. 8th Coll. QTDE, 2008 No. 14, p. 3 


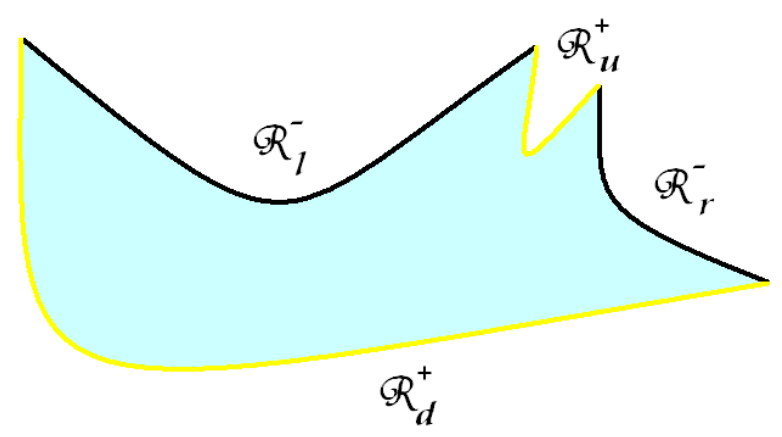

FiguRE 1. Example of an oriented rectangle embedded in the plane. The $[\cdot]^{-}$-components of the boundary are drawn with a thick dark line.

and it was also employed (in a more or less explicit form) by various authors in many different contexts $[7,9,30]$.

Lemma 2.1. [Crossing Lemma]. Let $\widetilde{\mathcal{R}}:=\left(\mathcal{R}, \mathcal{R}^{-}\right)$be an oriented rectangle in a metric space $X$ and suppose that $\mathcal{S} \subseteq \mathcal{R}$ is a compact set such that

$$
\mathcal{S} \cap \bar{\gamma} \neq \emptyset
$$

for each path $\gamma:[0,1] \rightarrow \mathcal{R}$ satisfying $\gamma(0) \in \mathcal{R}_{l}^{-}$and $\gamma(1) \in \mathcal{R}_{r}^{-}$. Then there exists a compact connected set $\mathcal{C} \subseteq \mathcal{S}$ such that

$$
\mathcal{C} \cap \mathcal{R}_{d}^{+} \neq \emptyset, \quad \mathcal{C} \cap \mathcal{R}_{u}^{+} \neq \emptyset .
$$

Proof. We give only a sketch of the proof. The missing details can be found in [45] or in [34, 35].

First of all, using the inverse of the homeomorphism $h: \mathcal{Q} \rightarrow \mathcal{R}$, we can confine ourselves to the study of a compact set $\mathcal{S} \subseteq \mathcal{Q}$ having the property of meeting any path $\gamma$ contained in $\mathcal{Q}$, with $\gamma$ joining the left and the right sides of $\mathcal{Q}$. If, by contradiction, we assume that $\mathcal{S}$ does not contain any compact connected set $\mathcal{C}$ intersecting both the lower and the upper sides of $\mathcal{Q}$, by the Whyburn Lemma (see [2]) we may find a decomposition of $\mathcal{S}$ into two disjoint compact subsets $A$ and $B$ EJQTDE, Proc. 8th Coll. QTDE, 2008 No. 14, p. 4 
such that

$$
\begin{aligned}
& A \cap([0,1] \times\{0\}) \neq \emptyset, \quad A \cap([0,1] \times\{1\})=\emptyset, \\
& B \cap([0,1] \times\{1\}) \neq \emptyset, \quad B \cap([0,1] \times\{0\})=\emptyset .
\end{aligned}
$$

The contradiction is now achieved by showing that there is a path $\sigma$ contained in $\mathcal{Q} \backslash \mathcal{S}$ and joining the left and the right sides of $\mathcal{Q}$ (see Figure 2). The existence of such a special path avoiding $\mathcal{S}=A \cup B$ may be proved by different techniques of topological or combinatorial nature (see, for instance [12, 20, 46] and [37] for a more detailed discussion of these and related results).

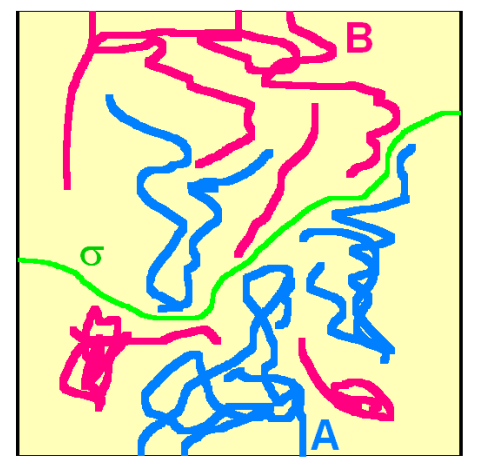

FiguRE 2. A pictorial explanation to the proof of Lemma 2.1.

The above Crossing Lemma may be used to give a simple proof of the Poincaré-Miranda theorem in dimension two. We recall that the Poincaré-Miranda theorem asserts the existence of a zero for a continuous vector field $F=\left(F_{1}, F_{2}\right)$ defined on a rectangle $\left[a_{1}, b_{1}\right] \times\left[a_{2}, b_{2}\right] \subseteq \mathbb{R}^{2}$ and such that $F_{1}\left(a_{1}, x_{2}\right) \leq 0 \leq F_{1}\left(b_{1}, x_{2}\right)$, for every $x_{2} \in\left[a_{2}, b_{2}\right]$ and $F_{2}\left(x_{1}, a_{2}\right) \leq 0 \leq F_{2}\left(x_{1}, b_{2}\right)$, for every $x_{1} \in\left[a_{1}, b_{1}\right]$. Actually, different combinations on the signs of $F_{1}$ and $F_{2}$ on the sides of the rectangle are allowed: the only crucial condition requires that the components of the vector field change their sign as the corresponding variables move from a side of the rectangle to the opposite one. The result holds also for the standard hypercube of $\mathbb{R}^{N}$ (as well as for $N$-dimensional rectangles). Such theorem is usually referred to Carlo Miranda who in [25] noted its equivalence to the Brouwer fixed-point theorem. On the other hand, as remarked in [23], Henry Poincaré in [41] and [42] had already EJQTDE, Proc. 8th Coll. QTDE, 2008 No. 14, p. 5 
announced this result with a suggestion of a correct proof using the Kronecker's index. In [42], with regard to the two-dimensional case, Poincaré (assuming strict inequalities for the components of the vector field on the boundary of the rectangle) described also an heuristic proof as follows: the "curve" $F_{2}=0$ departs from a point of the side $x_{1}=b_{1}$ and ends at some point of $x_{1}=a_{1}$; in the same manner, the curve $F_{1}=0$, departing from a point of $x_{2}=b_{2}$ and ending at some point of $x_{2}=a_{2}$, must necessarily meet the first "curve" in the interior of the rectangle. The interested reader may find in [19, 23] more information and historical remarks about the Poincaré-Miranda theorem.

Using Poincaré's heuristic argument, one could adapt his proof in the following manner: if we take any path $\gamma(t)$ contained in the rectangle $\left[a_{1}, b_{1}\right] \times\left[a_{2}, b_{2}\right]$ and joining the left and the right sides, by the Bolzano theorem we find at least a zero of $F_{1}(\gamma(t))$ and this, in turns, means that any path as above meets the set $\mathcal{S}:=F_{1}^{-1}(0)$. The Crossing Lemma implies the existence of a compact connected set $\mathcal{C}_{1} \subseteq F_{1}^{-1}(0)$ which intersects the lower and the upper sides of the rectangle. At this point one can easily achieve the conclusion in various different ways. For instance, one could just repeat the same argument on $F_{2}$ in order to obtain a compact connected set $\mathcal{C}_{2} \subseteq F_{2}^{-1}(0)$ which intersects the left and the right sides of the rectangle and thus prove the existence of a zero of the vector field $F$ using the fact that $\mathcal{C}_{1} \cap \mathcal{C}_{2} \neq \emptyset$. Alternatively, one could apply the Bolzano theorem and find a zero for $F_{2}$ restricted to $\mathcal{C}_{1}$ (see also [45] for a similar use of a variant of the Crossing Lemma and [40] for extensions to the $N$-dimensional setting). It may be interesting to observe that, conversely, it is possible to provide a proof of the Crossing Lemma via the Poincaré-Miranda theorem (see [35]).

Suppose that $\phi: X \supseteq D_{\phi} \rightarrow X$ is a map defined on a set $D_{\phi}$ and let $\widetilde{\mathcal{A}}:=\left(\mathcal{A}, \mathcal{A}^{-}\right)$and $\widetilde{\mathcal{B}}:=\left(\mathcal{B}, \mathcal{B}^{-}\right)$be oriented rectangles in a metric space $X$. Now we introduce the concept of "stretching along the paths" which plays a central role in our approach.

Definition 2.1. Let $\mathcal{H} \subseteq \mathcal{A} \cap D_{\phi}$ be a compact set. We say that $(\mathcal{H}, \phi)$ stretches $\widetilde{\mathcal{A}}$ to $\widetilde{\mathcal{B}}$ along the paths and write

$$
(\mathcal{H}, \phi): \widetilde{\mathcal{A}} \cong \widetilde{\mathcal{B}}
$$

if the following conditions hold:

- $\phi$ is continuous on $\mathcal{H}$;

EJQTDE, Proc. 8th Coll. QTDE, 2008 No. 14, p. 6 
- for every path $\gamma:[a, b] \rightarrow \mathcal{A}$ such that $\gamma(a) \in \mathcal{A}_{l}^{-}$and $\gamma(b) \in$ $\mathcal{A}_{r}^{-}$(or $\gamma(a) \in \mathcal{A}_{r}^{-}$and $\left.\gamma(b) \in \mathcal{A}_{l}^{-}\right)$, there exists a subinterval $\left[t^{\prime}, t^{\prime \prime}\right] \subseteq[a, b]$ such that

$$
\gamma(t) \in \mathcal{H}, \quad \phi(\gamma(t)) \in \mathcal{B}, \quad \forall t \in\left[t^{\prime}, t^{\prime \prime}\right]
$$

and, moreover, $\phi\left(\gamma\left(t^{\prime}\right)\right)$ and $\phi\left(\gamma\left(t^{\prime \prime}\right)\right)$ belong to different components of $\mathcal{B}^{-}$.

In the special case in which $\mathcal{H}=\mathcal{A}$, we simply write

$$
\phi: \widetilde{\mathcal{A}} \cong \widetilde{\mathcal{B}} \text {. }
$$

The definition of "crossing number" considered below is borrowed from Kennedy and Yorke [17] and adapted to our setting.

Definition 2.2. Let $\mathcal{D} \subseteq \mathcal{A} \cap D_{\phi}$ be a compact set and let $m \geq 2$ be an integer. We say that $(\mathcal{D}, \phi)$ stretches $\widetilde{\mathcal{A}}$ to $\widetilde{\mathcal{B}}$ along the paths with crossing number $m$ and write

$$
(\mathcal{D}, \phi): \widetilde{\mathcal{A}} \stackrel{m}{\underset{\sim}{\leftrightarrows}} \widetilde{\mathcal{B}}
$$

if there exist $m$ pairwise disjoint compact sets

$$
\mathcal{H}_{1}, \ldots, \mathcal{H}_{m} \subseteq \mathcal{D}
$$

such that

$$
\left(\mathcal{H}_{i}, \phi\right): \widetilde{\mathcal{A}} \cong \widetilde{\mathcal{B}}, \quad i=1, \ldots, m .
$$

In the special case in which $\mathcal{D}=\mathcal{A}$, we simply write

$$
\phi: \widetilde{\mathcal{A}} \stackrel{m}{\longleftarrow} \widetilde{\mathcal{B}} .
$$

Our first main result in this section concerns the existence and the localization of at least one fixed point for maps with the stretching property.

Theorem 2.1. Let $\phi: X \supseteq D_{\phi} \rightarrow X$ (with $X$ a metric space) be a map and $\widetilde{\mathcal{R}}=\left(\mathcal{R}, \mathcal{R}^{-}\right)$be an oriented rectangle with $\mathcal{R} \subseteq X$. Suppose that there is a compact set

$$
\mathcal{H} \subseteq \mathcal{R} \cap D_{\phi}
$$

such that

$$
(\mathcal{H}, \phi): \widetilde{\mathcal{R}} \cong \widetilde{\mathcal{R}} .
$$

Then there exists a point $w \in \mathcal{H}$ with $\phi(w)=w$.

EJQTDE, Proc. 8th Coll. QTDE, 2008 No. 14, p. 7 


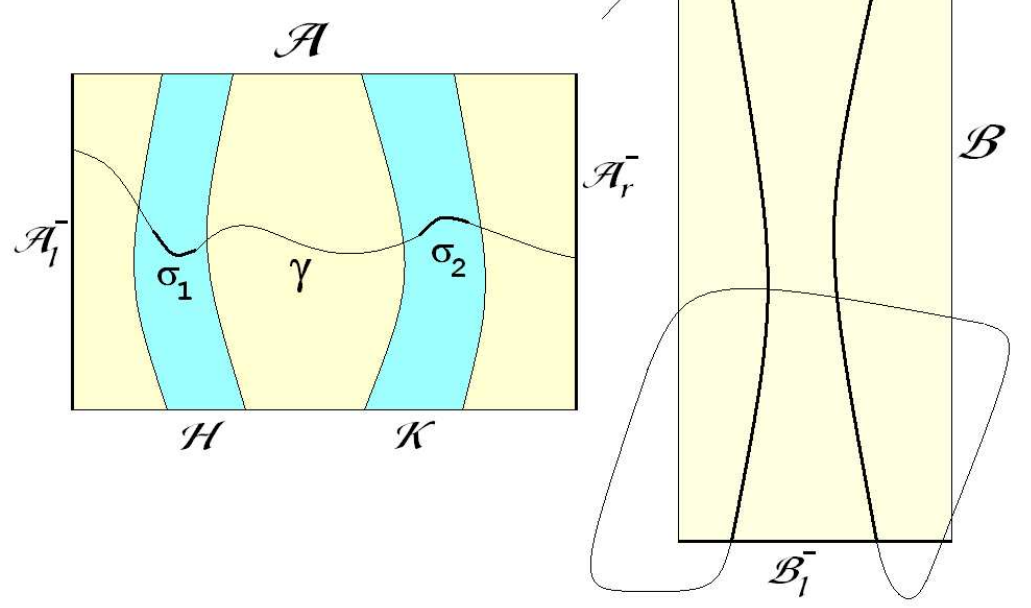

FiguRE 3. A visual explanation concerning Definition 2.1 and Definition 2.2: the rectangles $\mathcal{A}$ and $\mathcal{B}$, embedded in the plane, have been oriented by selecting, respectively, the sets $\mathcal{A}^{-}$and $\mathcal{B}^{-}$(drawn by thick lines). We have darkened two compact subsets $\mathcal{H}$ and $\mathcal{K}$ of $\mathcal{A}$. In the picture, we describe the action of a map $\phi$ such that $(\mathcal{H}, \phi): \widetilde{\mathcal{A}} \bumpeq \widetilde{\mathcal{B}}$ and also $(\mathcal{K}, \phi): \widetilde{\mathcal{A}} \cong \widetilde{\mathcal{B}}$. For a path $\gamma:[0,1] \rightarrow \mathcal{A}$ with $\gamma(0)$ and $\gamma(1)$ belonging to different components of $\mathcal{A}^{-}$, we have put in evidence two restrictions (sub-paths) $\sigma_{1}$ and $\sigma_{2}$ with range in $\mathcal{H}$ and $\mathcal{K}$, respectively, such that their composition with $\phi$ determines two new paths (drawn by bolder vertical lines) with values in $\mathcal{B}$ and connecting the two different components of $\mathcal{B}^{-}$. In this case, we could also write $\phi: \widetilde{\mathcal{A}} \stackrel{2}{\stackrel{2}{\sim}} \widetilde{\mathcal{B}}$.

Proof. Like in the proof of Lemma 2.1, it is convenient to reduce our problem to the unit square $\mathcal{Q}$, looking for a fixed point of the map $h^{-1} \circ \phi \circ h$. Actually, in order to avoid cumbersome notation, we assume (without loss of generality) $\mathcal{R}=\mathcal{Q}$ and

$$
(\mathcal{H}, \phi): \widetilde{\mathcal{Q}} \cong \widetilde{\mathcal{Q}}
$$

where we give the natural orientation to $\mathcal{Q}$, setting $\widetilde{\mathcal{Q}}=\left(\mathcal{Q}, \mathcal{Q}^{-}\right)$, with $\mathcal{Q}_{l}^{-}=\{0\} \times[0,1]$ and $\mathcal{Q}_{r}^{-}=\{1\} \times[0,1]$, as well as $\mathcal{Q}_{d}^{+}=[0,1] \times\{0\}$ and $\mathcal{Q}_{u}^{+}=[0,1] \times\{1\}$.

EJQTDE, Proc. 8th Coll. QTDE, 2008 No. 14, p. 8 
For $\phi=\left(\phi_{1}, \phi_{2}\right)$ and $x=\left(x_{1}, x_{2}\right)$, we define the set

$$
\mathcal{S}:=\left\{x \in \mathcal{H}: 0 \leq \phi_{2}(x) \leq 1, x_{1}-\phi_{1}(x)=0\right\},
$$

which is compact, as it is closed in $\mathcal{Q}$ (this follows from the continuity of $\phi$ on the closed set $\mathcal{H})$. Consider now a path $\gamma=\left(\gamma_{1}, \gamma_{2}\right):[0,1] \rightarrow \mathcal{Q}$ with $\gamma_{1}(0)=0$ and $\gamma_{1}(1)=1$. By the stretching assumption, there exists an interval $\left[t^{\prime}, t^{\prime \prime}\right] \subseteq[0,1]$ such that

$$
\gamma(t) \in \mathcal{H}, \quad \phi(\gamma(t)) \in \mathcal{Q}, \quad \forall t \in\left[t^{\prime}, t^{\prime \prime}\right]
$$

and $\phi_{1}\left(\gamma\left(t^{\prime}\right)\right)=0, \phi_{1}\left(\gamma\left(t^{\prime \prime}\right)\right)=1$, or $\phi_{1}\left(\gamma\left(t^{\prime}\right)\right)=1, \phi_{1}\left(\gamma\left(t^{\prime \prime}\right)\right)=0$. The continuous function $g(t):=\gamma_{1}(t)-\phi_{1}(\gamma(t))$, for $t \in\left[t^{\prime}, t^{\prime \prime}\right]$, satisfies $g\left(t^{\prime}\right) \geq 0 \geq g\left(t^{\prime \prime}\right)$, or $g\left(t^{\prime}\right) \leq 0 \leq g\left(t^{\prime \prime}\right)$, respectively, and therefore there exists $t^{*} \in\left[t^{\prime}, t^{\prime \prime}\right]$ with $\gamma\left(t^{*}\right) \in \mathcal{S}$. Then, by Lemma 2.1, there is a compact connected set $\mathcal{C} \subseteq \mathcal{S}$ such that $\mathcal{C} \cap \mathcal{Q}_{d}^{+} \neq \emptyset$ and $\mathcal{C} \cap \mathcal{Q}_{u}^{+} \neq \emptyset$. By definition of $\mathcal{S}$ we know that $\phi_{2}(w) \in[0,1], \forall w \in \mathcal{C}$. Hence, for every $P=\left(p_{1}, p_{2}\right) \in \mathcal{C} \cap \mathcal{Q}_{d}^{+}$we have $p_{2}-\phi_{2}(P) \leq 0$ and, similarly, $p_{2}-\phi_{2}(P) \geq 0$ for every $P=\left(p_{1}, p_{2}\right) \in \mathcal{C} \cap \mathcal{Q}_{u}^{+}$. Bolzano theorem guarantees the existence of at least a point $w=\left(w_{1}, w_{2}\right) \in \mathcal{C}$ such that $w_{2}-\phi_{2}(w)=0$. By the inclusion $\mathcal{H} \supseteq \mathcal{S} \supseteq \mathcal{C}$, we conclude also that $w_{1}-\phi_{1}(w)=0$ with $w \in \mathcal{H}$ and therefore $w$ is a fixed point for $\phi$ in $\mathcal{H}$.

The next step consists in proving the existence of infinitely many periodic points for a given map $\phi$. This is obtained by repeatedly applying Theorem 2.1 to the iterates of $\phi$ (see Theorem 2.2). To such end, we first observe that the stretching property is preserved under composition of maps. Indeed, we have:

Lemma 2.2. Let $\phi: X \supseteq D_{\phi} \rightarrow X$ and $\psi: X \supseteq D_{\psi} \rightarrow X$ (with $X$ a metric space) be such that

$$
(\mathcal{H}, \phi): \widetilde{\mathcal{A}} \cong \widetilde{\mathcal{B}} \text { and }(\mathcal{K}, \psi): \widetilde{\mathcal{B}} \bumpeq \widetilde{\mathcal{C}},
$$

where $\widetilde{\mathcal{A}}, \widetilde{\mathcal{B}}, \widetilde{\mathcal{C}}$ are oriented rectangles in $X$ and

$$
\mathcal{H} \subseteq \mathcal{A} \cap D_{\phi} \quad \text { and } \quad \mathcal{K} \subseteq \mathcal{B} \cap D_{\psi}
$$

are compact sets. Then

$$
(\mathcal{R}, \psi \circ \phi): \widetilde{\mathcal{A}} \cong \widetilde{\mathcal{C}}, \quad \text { for } \mathcal{R}:=\mathcal{H} \cap \phi^{-1}(\mathcal{K}) .
$$

EJQTDE, Proc. 8th Coll. QTDE, 2008 No. 14, p. 9 
The proof is a simple adaptation of that in [38, Lemma B.3, Appendix $\mathrm{B}]$ (where the case $X=\mathbb{R}^{2}$ was considered) and therefore it is omitted. By induction this lemma can be extended to the composition of an arbitrary number of maps.

Theorem 2.2. Let $\phi: X \supseteq D_{\phi} \rightarrow X$ be a map and $\widetilde{\mathcal{R}}=\left(\mathcal{R}, \mathcal{R}^{-}\right)$ an oriented rectangle of the metric space $X$. Suppose that there are a compact set $\mathcal{D} \subseteq \mathcal{R} \cap D_{\phi}$ and an integer $m \geq 2$ such that

$$
(\mathcal{D}, \phi): \widetilde{\mathcal{R}} \stackrel{m}{\stackrel{(}{\leftrightarrows}} \widetilde{\mathcal{R}} .
$$

Let also

$$
\mathcal{H}_{1}, \ldots, \mathcal{H}_{m} \subseteq \mathcal{D}
$$

be $m$ pairwise disjoint compact sets according to Definition 2.2. Then the following conclusions hold:

- for each $i=1, \ldots, m$, the map $\phi$ has at least a fixed point $w_{i} \in \mathcal{H}_{i}$

- let $\ell \geq 2$ and consider an arbitrary $\ell+1$-tuple $\left(s_{0}, s_{1}, \ldots, s_{\ell}\right)$ with $s_{i} \in\{1, \ldots, m\}$, for all $i=0, \ldots, \ell$ and such that $s_{0}=s_{\ell}$. Then there exists at least a point $w \in \mathcal{H}_{s_{0}}$ such that

$$
\phi^{(\ell)}(w)=w \quad \text { and } \phi^{(i)}(w) \in \mathcal{H}_{s_{i}}, \forall i=1, \ldots, \ell-1 .
$$

Proof. The existence of a fixed point for $\phi$ in each of the $\mathcal{H}_{i}$ 's follows from Theorem 2.1 and the fact that

$$
\left(\mathcal{H}_{i}, \phi\right): \widetilde{\mathcal{R}} \bumpeq \widetilde{\mathcal{R}}, \quad \forall i=1, \ldots, m .
$$

Consider now the second part of the statement. Let $\left(s_{0}, s_{1}, \ldots, s_{\ell}\right)$ be an arbitrary $\ell+1$-tuple (for $\ell \geq 2$ ) with $s_{i} \in\{1, \ldots, m\}$, for all $i=0, \ldots, \ell$ and such that $s_{0}=s_{\ell}$. Applying Lemma 2.2 to $\phi^{(\ell)}$, we have that

$$
\left(\mathcal{W}, \phi^{(\ell)}\right): \widetilde{\mathcal{R}} \cong \widetilde{\mathcal{R}}
$$

for the set

$$
\mathcal{W}:=\left\{x \in \mathcal{H}_{s_{0}}: \phi^{(i)}(x) \in \mathcal{H}_{s_{i}}, \forall i=1, \ldots, \ell\right\}
$$

and so Theorem 2.1 ensures the existence of a fixed point $w \in \mathcal{W}$ for $\phi^{(\ell)}$. The definition of $\mathcal{W}$ implies that $w \in \mathcal{H}_{s_{0}}$ and $\phi^{(i)}(w) \in \mathcal{H}_{s_{i}}$, for every $i=1, \ldots, \ell-1$. 
The result below gives information about the set of initial points which generate trajectories following an arbitrary forward itinerary. In the sequel, unless otherwise stated, sequences are one-sided and therefore are indexed on the set $\mathbb{N}$ of natural numbers. The two-sided sequences are indexed on $\mathbb{Z}$.

Theorem 2.3. Let $\phi: X \supseteq D_{\phi} \rightarrow X$ be a map and $\widetilde{\mathcal{R}}=\left(\mathcal{R}, \mathcal{R}^{-}\right)$ an oriented rectangle of a metric space $X$. Suppose that there are a compact set $\mathcal{D} \subseteq \mathcal{R} \cap D_{\phi}$ and an integer $m \geq 2$ such that

$$
(\mathcal{D}, \phi): \widetilde{\mathcal{R}} \stackrel{m}{\stackrel{m}{\sim}} \widetilde{\mathcal{R}} \text {. }
$$

Let also

$$
\mathcal{H}_{1}, \ldots, \mathcal{H}_{m} \subseteq \mathcal{D}
$$

be $m$ pairwise disjoint compact sets according to Definition 2.2. Then the following conclusions hold:

- for each sequence $\boldsymbol{s}=\left(s_{n}\right)_{n} \in\{1, \ldots, m\}^{\mathbb{N}}$ there exists a compact connected set $\mathcal{C}_{\boldsymbol{s}} \subseteq \mathcal{H}_{s_{0}}$ satisfying

$$
\mathcal{C}_{\boldsymbol{s}} \cap \mathcal{R}_{d}^{+} \neq \emptyset, \quad \mathcal{C}_{\boldsymbol{s}} \cap \mathcal{R}_{u}^{+} \neq \emptyset
$$

and such that $\phi^{(i)}(x) \in \mathcal{H}_{s_{i}}, \forall i \geq 1, \forall x \in \mathcal{C}_{\boldsymbol{s}}$;

- for each two-sided sequence $\left(s_{n}\right)_{n \in \mathbb{Z}} \in\{1, \ldots, m\}^{\mathbb{Z}}$ there exists a sequence of points $\left(x_{n}\right)_{n \in \mathbb{Z}}$ such that $\phi\left(x_{n-1}\right)=x_{n} \in \mathcal{H}_{s_{n}}, \forall n \in$ $\mathbb{Z}$.

Proof. We start by proving the first assertion. Let $\mathbf{s}=\left(s_{n}\right)_{n} \in\{1, \ldots, m\}^{\mathbb{N}}$ be an arbitrary sequence and define the set

$$
\mathcal{W}_{\mathbf{S}}:=\left\{z \in \mathcal{H}_{s_{0}}: \phi^{(i)}(z) \in \mathcal{H}_{s_{i}}, \forall i \geq 1\right\} .
$$

Let $\gamma_{0}:[0,1] \rightarrow \mathcal{R}$ be a path such that $\gamma_{0}(0)$ and $\gamma_{0}(1)$ belong to different components of $\mathcal{R}^{-}$. By the stretching assumption, there exists a subinterval

$$
\left[t_{1}^{\prime}, t_{1}^{\prime \prime}\right] \subseteq\left[t_{0}^{\prime}, t_{0}^{\prime \prime}\right]:=[0,1]
$$

such that

$$
\gamma_{0}(t) \in \mathcal{H}_{s_{0}} \quad \text { and } \quad \gamma_{1}(t):=\phi\left(\gamma_{0}(t)\right) \in \mathcal{R}, \quad \forall t \in\left[t_{1}^{\prime}, t_{1}^{\prime \prime}\right]
$$

By the same assumption, we also have that $\phi\left(\gamma_{0}\left(t_{1}^{\prime}\right)\right)$ and $\phi\left(\gamma_{0}\left(t_{1}^{\prime \prime}\right)\right)$ belong to different components of $\mathcal{R}^{-}$. Repeating inductively this argument, we produce a decreasing sequence of compact intervals

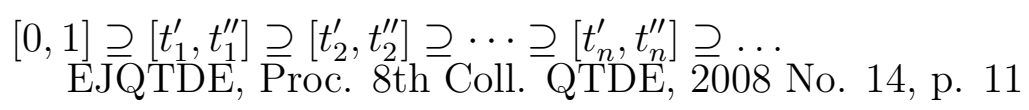


such that, setting

$$
\gamma_{n}(t):=\phi\left(\gamma_{n-1}(t)\right), \quad \forall t \in\left[t_{n}^{\prime}, t_{n}^{\prime \prime}\right], \quad n \geq 1,
$$

we have that, for every $n \geq 0$ :

$$
\gamma_{n}(t) \in \mathcal{R}, \quad \forall t \in\left[t_{n}^{\prime}, t_{n}^{\prime \prime}\right]
$$

$\gamma_{n}\left(t_{n}^{\prime}\right), \gamma_{n}\left(t_{n}^{\prime \prime}\right)$ belong to different components of $\mathcal{R}^{-}$and

$$
\gamma_{n}(t) \in \mathcal{H}_{s_{n}}, \quad \forall t \in\left[t_{n+1}^{\prime}, t_{n+1}^{\prime \prime}\right] .
$$

Hence we find that

$$
\gamma_{0}\left(t^{*}\right) \in \mathcal{W}_{\mathbf{S}} \quad \text { for any } t^{*} \in \bigcap_{n \geq 0}\left[t_{n}^{\prime}, t_{n}^{\prime \prime}\right] .
$$

This proves that any path in $\mathcal{R}$ joining the two components of $\mathcal{R}^{-}$ meets the set $\mathcal{W}_{\mathbf{S}}$ and therefore we can conclude by Lemma 2.1.

The second part of the statement (concerning two-sided sequences), follows from a diagonal argument already employed in a more general setting in [14, Proposition 5]. Therefore its proof is omitted (for more details, see also $[34,36])$.

Clearly, Theorem 2.2 and Theorem 2.3 indicate the presence of some kind of chaotic behaviour. To be more precise, we give now the explicit definition of chaos which will be used throughout the paper.

Definition 2.3. Let $X$ be a metric space, let $\phi: X \supseteq D_{\phi} \rightarrow X$ be a map and $\mathcal{D} \subseteq D_{\phi}$ a nonempty set. Let $m \geq 2$ be an integer. We say that $\phi$ induces chaotic dynamics on $m$ symbols in the set $\mathcal{D}$ if there exist $m$ nonempty pairwise disjoint compact sets

$$
\mathcal{H}_{1}, \mathcal{H}_{2}, \ldots, \mathcal{H}_{m} \subseteq \mathcal{D}
$$

such that, for each two-sided sequence $\left(s_{i}\right)_{i \in \mathbb{Z}} \in\{1, \ldots, m\}^{\mathbb{Z}}$, there exists a corresponding sequence $\left(w_{i}\right)_{i \in \mathbb{Z}} \in \mathcal{D}^{\mathbb{Z}}$ with

$$
w_{i} \in \mathcal{H}_{s_{i}} \quad \text { and } w_{i+1}=\phi\left(w_{i}\right), \quad \forall i \in \mathbb{Z}
$$

and, moreover, whenever $\left(s_{i}\right)_{i \in \mathbb{Z}}$ is a $k$-periodic sequence (that is, $\left.s_{i+k}=s_{i}, \forall i \in \mathbb{Z}\right)$ for some $k \geq 1$, there exists a $k$-periodic sequence $\left(w_{i}\right)_{i \in \mathbb{Z}} \in \mathcal{D}^{\mathbb{Z}}$ satisfying $(2.1)$.

\footnotetext{
${ }^{1}$ For $k=1$ this simply means that in each of the $\mathcal{H}_{i}$ 's there is at least a fixed point of $\phi$.
}

EJQTDE, Proc. 8th Coll. QTDE, 2008 No. 14, p. 12 
This definition (taken from [18] with minor variants) corresponds to a classical concept of chaos meant as the possibility of reproducing (via the iterates of a map) a general coin-flipping experiment [47, p. 42]. We also note that our definition, when applied to the case of a Poincaré map, agrees with other ones considered in the literature about chaotic dynamics for ODEs with periodic coefficients (see [8, 31, 49]). We further observe that a connection to the Bernoulli shift can be derived. With this respect, we first recall some basic results.

Let $m \geq 2$ be a positive integer. We denote by

$$
\Sigma_{m}=\{1, \ldots, m\}^{\mathbb{Z}}
$$

the set of the two-sided sequences of $m$ symbols. The set $\Sigma_{m}$ turns out to be a compact metric space under a standard metric $d$. For instance, a possible definition for such a metric is the following:

$$
d\left(\mathbf{s}^{\prime}, \mathbf{s}^{\prime \prime}\right):=\sum_{i \in \mathbb{Z}} \frac{\left|s_{i}^{\prime}-s_{i}^{\prime \prime}\right|}{m^{|i|}}, \quad \mathbf{s}^{\prime}=\left(s_{i}^{\prime}\right)_{i \in \mathbb{Z}}, \mathbf{s}^{\prime \prime}=\left(s_{i}^{\prime \prime}\right)_{i \in \mathbb{Z}} \in \Sigma_{m} .
$$

The Bernoulli shift $\sigma$ is the homeomorphism on $\Sigma_{m}$ defined by

$$
\sigma\left(\left(s_{i}\right)_{i}\right):=\left(s_{i+1}\right)_{i} .
$$

As proved in [1], $\sigma$ has positive topological entropy, given by

$$
h_{\mathrm{top}}(\sigma)=\log (m)
$$

Let $\Lambda$ be a compact metric space and let $\phi: \Lambda \rightarrow \Lambda$ be a continuous map. We say that $\phi$ is semiconjugate to the two-sided $m$-shift if there exists a continuous surjective mapping $g: \Lambda \rightarrow \Sigma_{m}$ such that

$$
g \circ \phi=\sigma \circ g .
$$

The following lemma relates the dynamical properties of a map satisfying Definition 2.3 to the ones of the Bernoulli shift.

Lemma 2.3. Let $X$ be a metric space, $\phi: X \supseteq D_{\phi} \rightarrow X$ be a map which is continuous and injective on a set $\mathcal{D} \subseteq D_{\phi}$ and induces therein chaotic dynamics on $m \geq 2$ symbols (relatively to $\left(\mathcal{H}_{1}, \ldots, \mathcal{H}_{m}\right)$ ). Then, there exists a nonempty compact set

$$
\Lambda \subseteq \bigcup_{j=1}^{m} \mathcal{H}_{j}
$$

EJQTDE, Proc. 8th Coll. QTDE, 2008 No. 14, p. 13 
with

$$
\phi(\Lambda)=\Lambda
$$

and such that $\left.\phi\right|_{\Lambda}$ is semiconjugate to the two-sided $m$-shift. Moreover, the subset $\mathcal{P}$ of $\Lambda$ made by the periodic points of $\phi$ is dense in $\Lambda$ and if we denote by $g: \Lambda \rightarrow \Sigma_{m}$ the continuous surjection in (2.2), it holds also that the counterimage through $g$ of any $k$-periodic sequence in $\Sigma_{m}$ contains at least one $k$-periodic point of $\phi$.

The proof, which is based on arguments presented for instance in [14, 17], is omitted. Complete details can be found in [40]. We observe that the existence of a semiconjugation to the shift map on $\Sigma_{m}$ implies that the topological entropy of $\left.\phi\right|_{\Lambda}$ satisfies

$$
h_{\text {top }}\left(\left.\phi\right|_{\Lambda}\right) \geq \log (m)
$$

(see [53]).

Now we are in position to reconsider Theorem 2.2 and Theorem 2.3 in order to obtain the following:

Theorem 2.4. Let $\phi: X \supseteq D_{\phi} \rightarrow X$ be a map and $\widetilde{\mathcal{R}}=\left(\mathcal{R}, \mathcal{R}^{-}\right)$ an oriented rectangle of a metric space $X$. Suppose that there are a compact set $\mathcal{D} \subseteq \mathcal{R} \cap D_{\phi}$ and an integer $m \geq 2$ such that

$$
(\mathcal{D}, \phi): \widetilde{\mathcal{R}} \stackrel{m}{\sim} \widetilde{\mathcal{R}}
$$

Let also

$$
\mathcal{H}_{1}, \ldots, \mathcal{H}_{m} \subseteq \mathcal{D}
$$

be $m$ pairwise disjoint compact sets according to Definition 2.2. Then the map $\phi$ induces chaotic dynamics on $m$ symbols in the set

$$
\mathcal{H}:=\bigcup_{i=1}^{m} \mathcal{H}_{i} \subseteq \mathcal{D} .
$$

Moreover, for each sequence $s=\left(s_{n}\right)_{n} \in\{1, \ldots, m\}^{\mathbb{N}}$, there exists a compact connected set $\mathcal{C}_{s} \subseteq \mathcal{H}_{s_{0}}$ with

$$
\mathcal{C}_{s} \cap \mathcal{R}_{d}^{+} \neq \emptyset, \quad \mathcal{C}_{s} \cap \mathcal{R}_{u}^{+} \neq \emptyset
$$

and such that, for every $w \in \mathcal{C}_{s}$,

$$
\phi^{(n)}(w) \in \mathcal{H}_{s_{n}}, \quad \forall n \geq 1 .
$$

EJQTDE, Proc. 8th Coll. QTDE, 2008 No. 14, p. 14 
In our applications the function $\phi$ will the Poincaré map

$$
\phi: z \mapsto u(T, z)
$$

associated to a $T$-periodic non-autonomous second order ODE (with $u(\cdot, z)$ the solution of a given ODE satisfying the initial condition $u(0)=z)$. In this case, the relation $\phi^{(n)}(z) \in \mathcal{H}_{i}$ will be interpreted in terms of the oscillatory behaviour of the solution $u(\cdot, z)$ along the time interval $[n T,(n+1) T]$.

\section{A CONNECTION TO LINKED TWIST MAPS}

In the past decades a growing interest has concerned the so-called "Linked Twist Maps" (shortly LTMs). Such kind of maps furnish a geometrical setting for the existence of Smale horseshoes and appear in a natural manner in various different applicative contexts. Around the '80s they were studied from a theoretical point of view by Devaney [10], Burton and Easton [6] and Przytycki [43, 44] (just to cite a few contributions in this direction), proving some mathematical properties like ergodicity, hyperbolicity and conjugation to the Bernoulli shift. Special configurations related to LTMs appear in the restricted three-body problem [28, pp. 90-94], as well as in the study of diffeomorphisms of surfaces or in mathematical models for particle motions in a magnetic field (see the corresponding references in [10]). More recently, in the work of Ottino, Sturman and Wiggins (see, for instance, [50, 54, 55]), LTMs have found applications in the study of fluid mixing. Up to a homeomorphism (or a diffeomorphism), such maps are defined on the union of two circular annuli (or two families of them [44]) intersecting in two different regions. In each annulus a continuous twist mapping is defined, that is, a map which leaves the annulus invariant and rotates its inner and outer boundaries at different angular speeds. Therefore it is possible to consider the composition of the two movements in the common regions: the resulting function is what we call a linked twist map (see $[54,55]$ for a detailed description of the geometry of the domain of a LTM). Usual assumptions on the twist mappings involved in the composition require, among others, their smoothness, monotonicity of the angular speed with respect to the radial coordinate and preservation of the Lebesgue measure. In our approach (which is purely topological), we'll just need a twist condition on the boundary.

Our purpose now is to adapt the general results of Section 2 to a geometrical situation which is connected to and generalizes the case of EJQTDE, Proc. 8th Coll. QTDE, 2008 No. 14, p. 15 
the linked twist maps. The corresponding theorem (see Theorem 3.1 below) will be then applied to some examples of nonlinear ODEs with periodic coefficients.

Theorem 3.1. Let $X$ be a metric space, let $f: X \supseteq D_{f} \rightarrow X$ and $g$ : $X \supseteq D_{g} \rightarrow X$ be continuous maps and let $\widetilde{\mathcal{P}}:=\left(\mathcal{P}, \mathcal{P}^{-}\right), \widetilde{\mathcal{O}}:=\left(\mathcal{O}, \mathcal{O}^{-}\right)$ be oriented rectangles in $X$. Suppose that the following conditions are satisfied:

$\left(H_{f}\right) \quad$ there exist a compact set $\mathcal{D} \subseteq \mathcal{P} \cap D_{f}$ and an integer $m \geq 2$

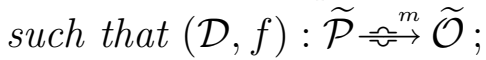

$\left(H_{g}\right) \quad \mathcal{O} \subseteq D_{g}$ and $g: \widetilde{\mathcal{O}} \cong \widetilde{\mathcal{P}}$.

Let also $\mathcal{H}_{1}, \ldots, \mathcal{H}_{m} \subseteq \mathcal{D}$ be $m$ pairwise disjoint compact sets such that $\left(\mathcal{H}_{i}, f\right): \widetilde{\mathcal{P}} \bumpeq \widetilde{\mathcal{O}}$, for $i=1, \ldots, m$.

Then the map $\phi:=g \circ f$ induces chaotic dynamics on $m$ symbols in the set $\mathcal{H}:=\cup_{i=1}^{m} \mathcal{H}_{i} \subseteq \mathcal{D}$. Moreover, for each sequence of $m$ symbols $s=\left(s_{n}\right)_{n} \in\{1, \ldots, m\}^{\mathbb{N}}$, there exists a compact connected set $\mathcal{C}_{s} \subseteq \mathcal{H}_{s_{0}}$ with

$$
\mathcal{C}_{s} \cap \mathcal{P}_{d}^{+} \neq \emptyset, \quad \mathcal{C}_{s} \cap \mathcal{P}_{u}^{+} \neq \emptyset
$$

and such that, for every $w \in \mathcal{C}_{s}$ there exists a sequence $\left(y_{n}\right)_{n}$ with $y_{0}=w$ and

$$
y_{n} \in \mathcal{H}_{s_{n}}, \quad \phi\left(y_{n}\right)=y_{n+1}, \forall n \geq 0 .
$$

Proof. We prove

$$
\left(\mathcal{H}_{i}, \phi\right): \widetilde{\mathcal{P}} \cong \widetilde{\mathcal{P}}, \quad \forall i=1, \ldots, m
$$

from which the conclusion immediately follows by Theorem 2.4. To check condition (3.1), let us consider a path $\gamma:[0,1] \rightarrow \mathcal{P}$ such that $\gamma(0) \in \mathcal{P}_{l}^{-}$and $\gamma(1) \in \mathcal{P}_{r}^{-}$and let $i \in\{1, \ldots, m\}$ be fixed. By $\left(H_{f}\right)$, there exists a compact interval $\left[t_{1}, t_{2}\right] \subseteq[0,1]$ such that $\gamma(t) \in \mathcal{H}_{i}$ and $f(\gamma(t)) \in \mathcal{O}$ for every $t \in\left[t_{1}, t_{2}\right]$. We have also that $f\left(\gamma\left(t_{1}\right)\right)$ and $f\left(\gamma\left(t_{2}\right)\right)$ belong to different components of $\mathcal{O}^{-}$. Just to fix a case for our discussion (the other one is completely analogous), we suppose that

$$
f\left(\gamma\left(t_{1}\right)\right) \in \mathcal{O}_{l}^{-}, \quad f\left(\gamma\left(t_{2}\right)\right) \in \mathcal{O}_{r}^{-} .
$$

Define now

$$
\sigma(t):=f(\gamma(t)), \forall t \in\left[t_{1}, t_{2}\right]
$$

$\sigma:\left[t_{1}, t_{2}\right] \rightarrow \mathcal{O}$. By $\left(H_{g}\right)$ there exists a compact interval $\left[t^{\prime}, t^{\prime \prime}\right] \subseteq\left[t_{1}, t_{2}\right]$ such that $g(\sigma(t)) \in \mathcal{P}$ for every $t \in\left[t^{\prime}, t^{\prime \prime}\right]$ and also $g\left(\sigma\left(t^{\prime}\right)\right)$ and $g\left(\sigma\left(t^{\prime \prime}\right)\right)$ EJQTDE, Proc. 8th Coll. QTDE, 2008 No. 14, p. 16 
belong to different components of $\mathcal{P}^{-}$.

We have thus showed that

$$
\gamma(t) \in \mathcal{H}_{i} \quad \text { and } \phi(\gamma(t)) \in \mathcal{P}, \quad \forall t \in\left[t^{\prime}, t^{\prime \prime}\right]
$$

with $\phi\left(\gamma\left(t^{\prime}\right)\right)$ and $\phi\left(\gamma\left(t^{\prime \prime}\right)\right)$ belonging to different components of $\mathcal{P}^{-}$. This concludes the proof of (3.1) and also of the theorem.

We present now two simple examples for the application of Theorem 3.1 which show how this result is well-fit for studying LTMs. The first of the two examples is fairly classical as it concerns two overlapping annuli under twist rotations. The second one describes the composition of a map which twists an annulus with a longitudinal motion along a strip. Our examples are only of "pedagogical" nature and, in fact, the chaotic-like dynamics that we obtain therein could be also proved using different approaches already developed by various authors (like, for instance, in $[27,48,56,60])$.

Example 3.1. A classical example of a LTM is represented by the composition of two planar maps $f_{1}$ and $f_{2}$ which act as twist rotations around two given points. For instance, a possible choice is that of considering two maps expressed by means of complex variables as

$$
f_{1}(z):=-r+(z+r) \exp \left(\imath\left(e_{1}+a_{1}|z+r|\right)\right)
$$

and

$$
f_{2}(z):=r+(z-r) \exp \left(\imath\left(e_{2}+a_{2}|z-r|\right)\right),
$$

where $\imath$ is the imaginary unit, while $r>0, a_{j} \neq 0$ and $e_{j}(j=1,2)$ are real coefficients. Such maps twist around the centers $(-r, 0)$ and $(r, 0)$, respectively. We denote by $p_{1}$ and $p_{2}$ the inner and the outer radii for the annulus around $(-r, 0)$ and by $q_{1}$ and $q_{2}$ the inner and the outer radii for the annulus around $(r, 0)$.

For a suitable choice of $r$, of the radii $p_{1}<p_{2}$ and $q_{1}<q_{2}$, as well as of the parameters determining $f_{1}$ and $f_{2}$, it is possible to apply Theorem 3.1 in order to obtain chaotic dynamics (see Fig. 4-5).

Example 3.2. A different case of a nonstandard LTM is represented by the composition of two planar maps $f_{1}$ and $f_{2}$, where $f_{1}$ is a twist rotation around a given point while $f_{2}$ produces a longitudinal motion along a strip with different velocities on the upper and lower components of the boundary of the strip. To present a possible example in EJQTDE, Proc. 8th Coll. QTDE, 2008 No. 14, p. 17 


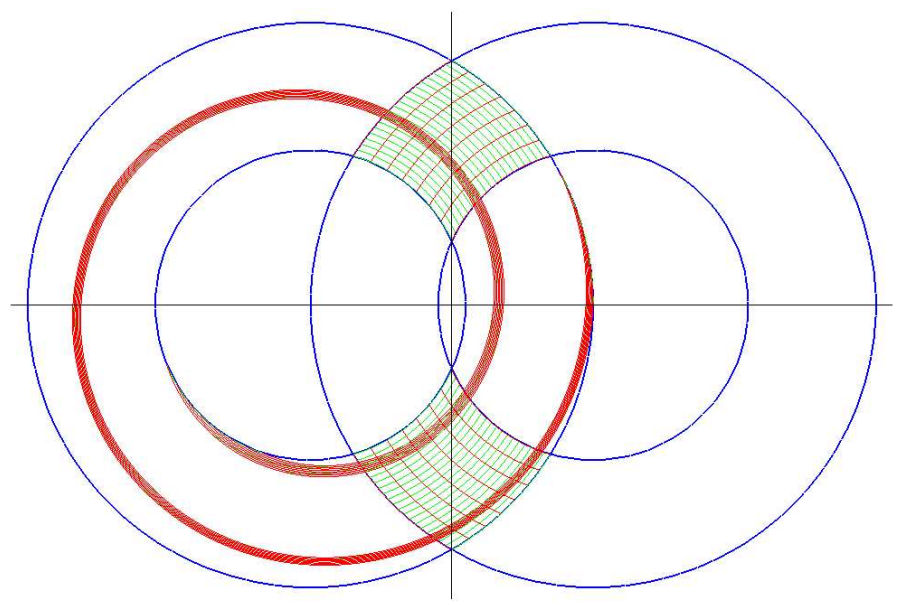

FIgURE 4. A pictorial comment to condition $\left(H_{f}\right)$ of Theorem 3.1, with reference to Example 3.1. We have chosen $r=3$ and $p_{1}=q_{1}=3.3, p_{2}=q_{2}=6$ in order to determine the two annuli (this is just to simplify the example, since no special symmetry is needed). For the map $f_{1}$ we have taken $a_{1}=3.3$ and $e_{1}=-1.5$. We define the set $\mathcal{P}$ of Theorem 3.1 as the upper intersection of the two annuli and select the two components of $\mathcal{P}^{-}$as the intersections of $\mathcal{P}$ with the inner and outer boundaries of the annulus at the left hand side. The set $\mathcal{O}$ is defined as the the lower intersection of the two annuli and the two components of $\mathcal{O}^{-}$are the intersections of $\mathcal{O}$ with the inner and outer boundaries of the annulus at the right hand side. The narrow strip, spiralling inside the left annulus, is the image of $\mathcal{P}$ under $f_{1}$. Clearly, any path in $\mathcal{P}$ joining the two components of $\mathcal{P}^{-}$is transformed by $f_{1}$ onto a path crossing $\mathcal{O}$ twice in the correct manner.

this direction, we first introduce, for any pair of real numbers $a<b$, the real valued function

$$
\operatorname{Pr}_{[a, b]}(t):=\frac{1}{b-a} \min \{b-a, \max \{0, t-a\}\},
$$

and then we define

$$
g_{1}(t):=e_{1}+c_{1} \operatorname{Pr}_{\left[p_{1}, p_{2}\right]}(t), \quad g_{2}(t):=e_{2}+c_{2} \operatorname{Pr}_{\left[q_{1}, q_{2}\right]}(t),
$$

EJQTDE, Proc. 8th Coll. QTDE, 2008 No. 14, p. 18 


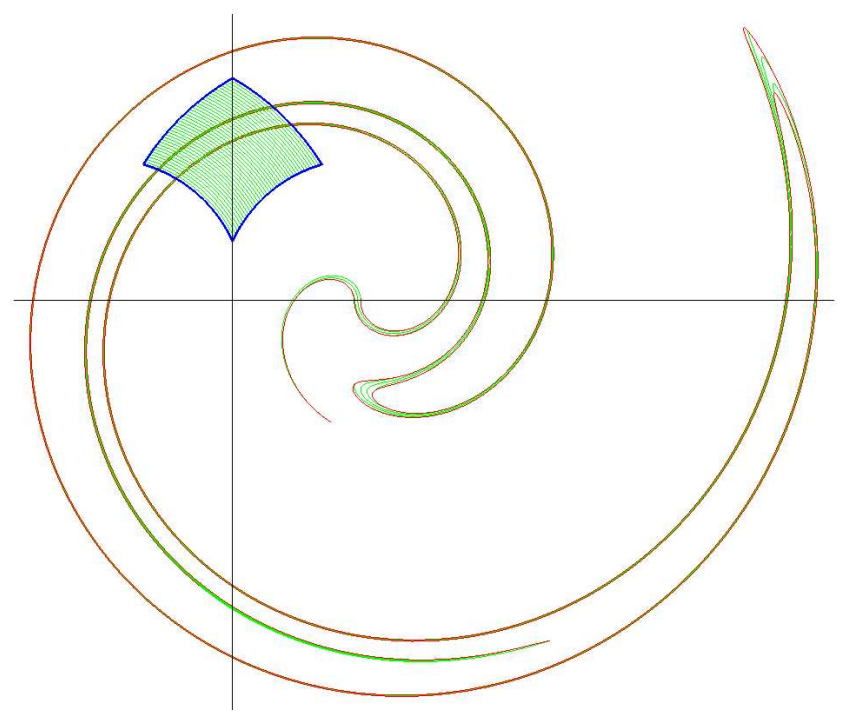

FiguRE 5. For $r, p_{1}, p_{2}, q_{1}, q_{2}$ and $f_{1}$ as in Fig. 4, we have also fixed the parameters of $f_{2}$ by taking $a_{2}=0.9$ and $e_{2}=$ 0 . The present picture shows the image of $\mathcal{P}$ through the composite map $f_{2} \circ f_{1}$. It is evident that $\left(f_{2} \circ f_{1}\right)(\mathcal{P})$ crosses $\mathcal{P}$ twice in the correct way.

where $c_{j} \neq 0$ and $e_{j}(j=1,2)$ are real coefficients and

$$
0<p_{1}<p_{2} \text { and }-p_{1}<q_{1}<q_{2}<p_{1}
$$

are some real parameters which determine, respectively, the inner and the outer radii of the annulus

$$
A\left[p_{1}, p_{2}\right]:=\left\{(x, y): p_{1}^{2} \leq x^{2}+y^{2} \leq p_{2}^{2}\right\}
$$

and the position of the strip

$$
S\left[q_{1}, q_{2}\right]:=\left\{(x, y): q_{1} \leq y \leq q_{2}\right\} .
$$

Now we define two maps expressed by means of complex variables as

$$
f_{1}(z):=z \exp \left(\imath g_{1}(|z|)\right)
$$

(which twists around the origin) and, for $\Im(z)=\frac{z-\bar{z}}{2 \imath}$,

$$
f_{2}(z):=z+g_{2}(\Im(z))
$$

(which shifts the points along the horizontal lines).

For a suitable choice of the parameters determining $f_{1}$ and $f_{2}$, it is EJQTDE, Proc. 8th Coll. QTDE, 2008 No. 14, p. 19 
possible to apply Theorem 3.1 in order to obtain chaotic dynamics (see Fig. 6-7-8).

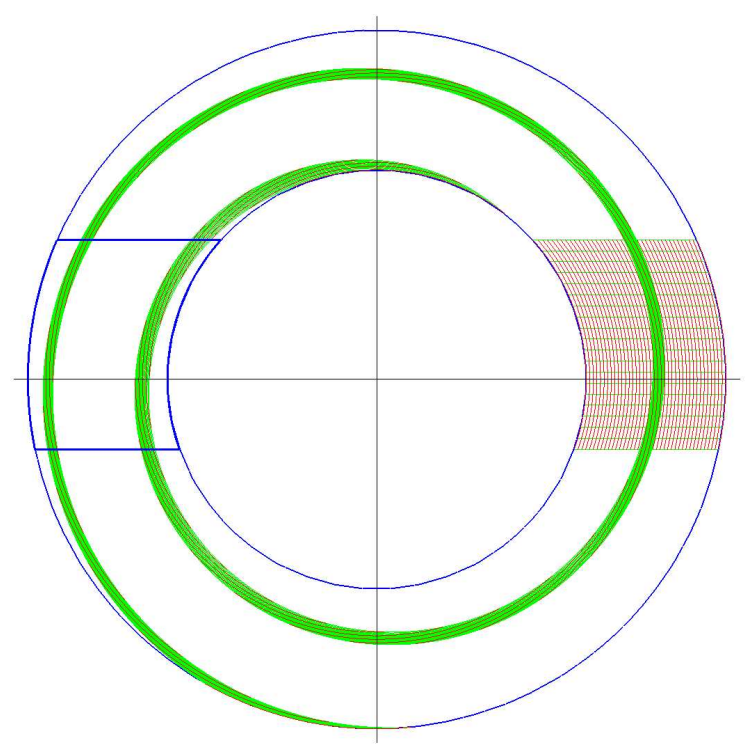

FiguRE 6. A pictorial comment to condition $\left(H_{f}\right)$ of Theorem 3.1, with reference to Example 3.2. We have chosen $p_{1}=3, p_{2}=5, q_{1}=-1$ and $q_{2}=2$ in order to determine the annulus $A\left[p_{1}, p_{2}\right]$ centered at the origin and the strip $S\left[q_{1}, q_{2}\right]$ which goes across it. For the map $f_{1}$ we have taken $e_{1}=0.4 \pi$ and $c_{1}=3 \pi$. We define the set $\mathcal{P}$ of Theorem 3.1 as the right hand side intersection of the annulus with the strip and select the two components of $\mathcal{P}^{-}$as the intersections of $\mathcal{P}$ with the inner and outer boundaries of the annulus. The set $\mathcal{O}$ is defined as the left hand side intersection of the annulus with the strip and the two components of $\mathcal{O}^{-}$are the intersections of $\mathcal{O}$ with the lower and upper sides of the strip. The narrow band, spiralling inside the annulus, is the image of $\mathcal{P}$ under $f_{1}$. Clearly, any path in $\mathcal{P}$ joining the two components of $\mathcal{P}^{-}$is transformed by $f_{1}$ onto a path crossing $\mathcal{O}$ twice in the correct manner.

With a straightforward modification in the proof of Theorem 3.1, one could verify that $\left(H_{f}\right)$ and $\left(H_{g}\right)$ imply the existence of chaotic dynamics also for the map $f \circ g$. Actually, both Theorem 3.1 and such a variant of it can be obtained as corollaries of the following more general result that we state without proof.

EJQTDE, Proc. 8th Coll. QTDE, 2008 No. 14, p. 20 


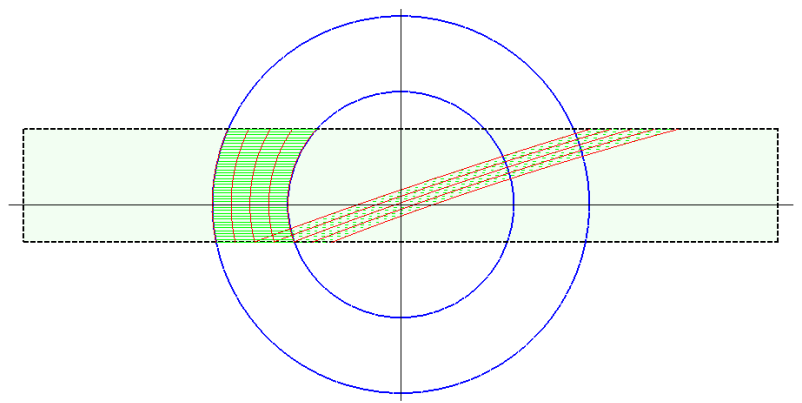

Figure 7. A pictorial comment to condition $\left(H_{g}\right)$ of Theorem 3.1, with reference to Example 3.2. We have chosen $A\left[p_{1}, p_{2}\right]$ and $S\left[q_{1}, q_{2}\right]$ with $p_{1}, p_{2}, q_{1}, q_{2}$ as in Fig. 6 and defined the oriented rectangles $\widetilde{\mathcal{P}}=\left(\mathcal{P}, \mathcal{P}^{-}\right), \widetilde{\mathcal{O}}=\left(\mathcal{O}, \mathcal{O}^{-}\right)$ also as before. For the map $f_{2}$ we have taken $e_{2}=1$ and $c_{2}=8.6$. The parallelogram-shaped narrow figure inside the strip $S\left[q_{1}, q_{2}\right]$ is the image of $\mathcal{O}$ under $f_{2}$. Clearly, any path in $\mathcal{O}$ joining the two components of $\mathcal{O}^{-}$is transformed by $f_{2}$ to a path crossing $\mathcal{P}$ once in the right way.

Theorem 3.2. Let $X$ be a metric space, let $\varphi: X \supseteq D_{\varphi} \rightarrow X$ and $\psi$ : $X \supseteq D_{\psi} \rightarrow X$ be continuous maps and let $\widetilde{\mathcal{A}}:=\left(\mathcal{A}, \mathcal{A}^{-}\right), \widetilde{\mathcal{B}}:=\left(\mathcal{B}, \mathcal{B}^{-}\right)$ be oriented rectangles in $X$. Suppose that the following conditions are satisfied:

$\left(H_{\varphi}\right)$ there exist $m \geq 1$ pairwise disjoint compact sets $\mathcal{H}_{1}, \ldots, \mathcal{H}_{m} \subseteq$ $\mathcal{A} \cap D_{\varphi}$ such that $\left(\mathcal{H}_{i}, \varphi\right): \widetilde{\mathcal{A}} \bumpeq \widetilde{\mathcal{B}}$, for $i=1, \ldots, m$;

$\left(H_{\psi}\right)$ there exist $\ell \geq 1$ pairwise disjoint compact sets $\mathcal{K}_{1}, \ldots, \mathcal{K}_{\ell} \subseteq$ $\mathcal{B} \cap D_{\psi}$ such that $\left(\mathcal{K}_{i}, \psi\right): \widetilde{\mathcal{B}} \bumpeq \widetilde{\mathcal{A}}$, for $i=1, \ldots, \ell$.

If at least one between $m$ and $\ell$ is greater or equal than 2 , then the composite map $\phi:=\psi \circ \varphi$ induces chaotic dynamics on $m \times \ell$ symbols EJQTDE, Proc. 8th Coll. QTDE, 2008 No. 14, p. 21 


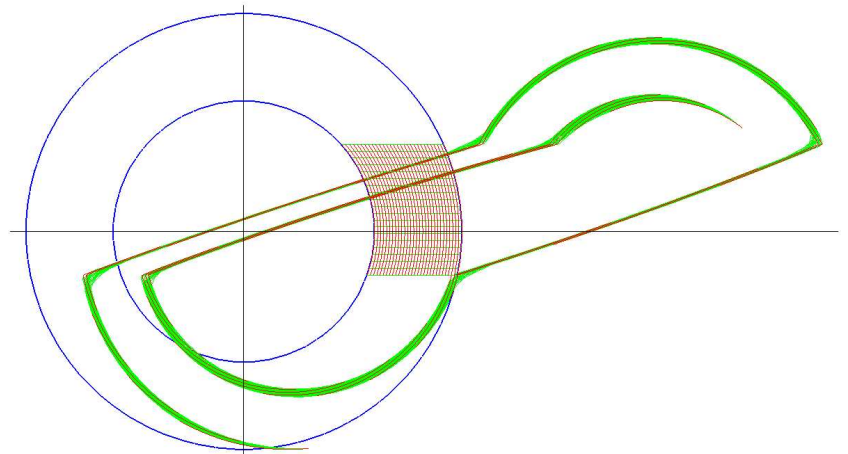

Figure 8. For $p_{1}, p_{2}, q_{1}, q_{2}$ and $f_{1}$ as in Fig. 6 and $f_{2}$ as in Fig. 7, we show the image of $\mathcal{P}$ through the composite map $f_{2} \circ f_{1}$. It is evident that $\left(f_{2} \circ f_{1}\right)(\mathcal{P})$ crosses $\mathcal{P}$ twice in the correct manner.

in the set

$$
\mathcal{H}^{*}:=\bigcup_{\substack{i=1, \ldots, m \\ j=1, \ldots, \ell}} \mathcal{H}_{i, j}^{\prime} \quad \text { with } \quad \mathcal{H}_{i, j}^{\prime}:=\mathcal{H}_{i} \cap \varphi^{-1}\left(\mathcal{K}_{j}\right)
$$

Moreover, for each sequence of $m \times \ell$ symbols

$$
\boldsymbol{s}=\left(s_{n}\right)_{n}=\left(p_{n}, q_{n}\right)_{n} \in\{1, \ldots, m\}^{\mathbb{N}} \times\{1, \ldots, \ell\}^{\mathbb{N}},
$$

there exists a compact connected set $\mathcal{C}_{s} \subseteq \mathcal{H}_{p_{0}, q_{0}}^{\prime}$ with

$$
\mathcal{C}_{s} \cap \mathcal{A}_{d}^{+} \neq \emptyset, \quad \mathcal{C}_{s} \cap \mathcal{A}_{u}^{+} \neq \emptyset
$$

and such that, for every $w \in \mathcal{C}_{s}$, there exists a sequence $\left(y_{n}\right)_{n}$ with $y_{0}=w$ and

$y_{n} \in \mathcal{H}_{p_{n}, q_{n}}^{\prime}, \quad \phi\left(y_{n}\right)=y_{n+1}, \forall n \geq 0$.

EJQTDE, Proc. 8th Coll. QTDE, 2008 No. 14, p. 22 


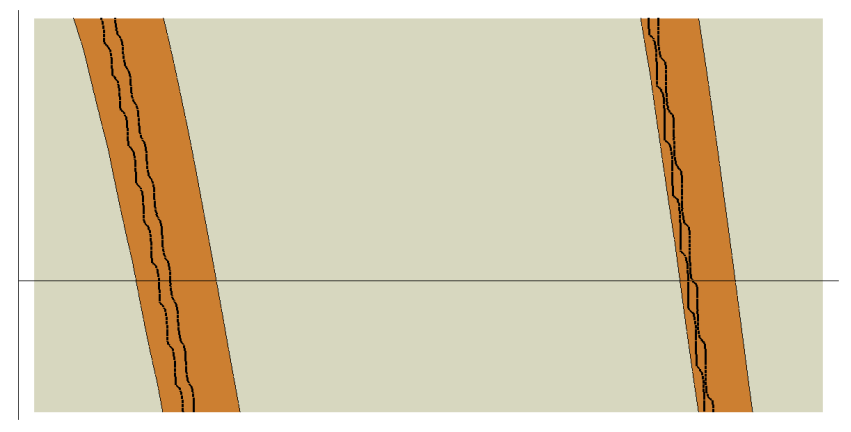

Figure 9. In the simple case of Example 3.2 and for $f_{1}$ and $f_{2}$ determined by the coefficients chosen for drawing Fig. 8, we can determine the sets $\mathcal{H}_{1}$ and $\mathcal{H}_{2}$ in Theorem 3.1. Indeed, in the set $\mathcal{P}$, transformed in the rectangle $\left[p_{1}, p_{2}\right] \times$ $\left[q_{1}, q_{2}\right]$ by a suitable change of coordinate, we have found two subsets (drawn with a darker color) whose image through $f_{1}$ are contained in $\mathcal{O}$. Inside these subsets, we have found two smaller domains such that their images through $f_{2} \circ f_{1}$ are contained again in $\mathcal{P}$. Clearly, any path contained in the rectangle $\left[p_{1}, p_{2}\right] \times\left[q_{1}, q_{2}\right]$ and joining the left to the right sides contains two sub-paths which are stretched by the composite mapping to paths connecting again the left and the right sides. Up to a homeomorphism between $\mathcal{P}$ and $\left[p_{1}, p_{2}\right] \times$ $\left[q_{1}, q_{2}\right]$, this is precisely what happens in $\left(\mathcal{P}, \mathcal{P}^{-}\right)$for $f_{2} \circ f_{1}$.

\section{An APplication to SECOND ORDER ODEs}

In this section we show a possible application of Theorem 3.1 to the second order scalar ODE

$$
x^{\prime \prime}+f(t, x)=0,
$$

where $f: \mathbb{R} \times \mathbb{R} \rightarrow \mathbb{R}$ satisfies the Carathéodory assumptions and is $T$-periodic in the $t$-variable.

An important subclass of equation (4.1) is given by the periodically perturbed Duffing equations

$$
x^{\prime \prime}+g(x)=p(t),
$$

where $g: \mathbb{R} \rightarrow \mathbb{R}$ is a locally Lipschitzean function and $p(\cdot): \mathbb{R} \rightarrow \mathbb{R}$ is a locally integrable function such that $p(t+T)=p(t)$, for almost every $t \in \mathbb{R}$ (for some $T>0$ ).

In [38] a result on the existence of infinitely many periodic solutions, EJQTDE, Proc. 8th Coll. QTDE, 2008 No. 14, p. 23 
as well as of complex dynamics, has been obtained for a special case of equation (4.2). More precisely, in [38] the equation

$$
x^{\prime \prime}+k x^{+}=p_{r, s}(t)
$$

has been considered, by assuming that $k, r, s, \tau_{r}, \tau_{s}$ are positive real numbers and $p_{r, s}: \mathbb{R} \rightarrow \mathbb{R}$ is a periodic function of period

$$
T=T_{r, s}:=\tau_{r}+\tau_{s},
$$

defined on $[0, T[$ by

$$
p_{r, s}(t):=\left\{\begin{aligned}
r, & \text { for } 0 \leq t<\tau_{r} \\
-s, & \text { for } \tau_{r} \leq t<\tau_{r}+\tau_{s},
\end{aligned}\right.
$$

and then extended to the real line by $T$-periodicity.

Equation (4.3) can be regarded as a simplified version of the LazerMcKenna suspension bridges model (see $[21,22]$ ). It is also meaningful from the point of view of the study of ODEs with "jumping nonlinearities" and the periodic Dancer-Fučik spectrum (see [24] for a very interesting survey concerning recent results in that area).

Our main result is the following:

Theorem 4.1. For any choice of $k, r, s>0$ and any positive integer $m \geq 2$, it is possible to find two intervals $] a_{r}^{m}, b_{r}^{m}[$ and $] a_{s}^{m}, b_{s}^{m}[$ such that, for every forcing term $p_{r, s}$ with $\left.\tau_{r} \in\right] a_{r}^{m}, b_{r}^{m}\left[\right.$ and $\left.\tau_{s} \in\right] a_{s}^{m}, b_{s}^{m}[$, the Poincaré map associated to (4.3) induces chaotic dynamics on $m$ symbols in the plane.

In [38, Theorem 1.2], precise information about the oscillatory behaviour of the solutions is given too. The above result is stable with respect to small perturbations in the sense that once we have proved the existence of chaotic-like dynamics in a certain range of parameters for equation (4.3), then the same result still holds for

$$
x^{\prime \prime}+c x^{\prime}+k x^{+}=p(t)
$$

provided that $|c|$ and $\int_{0}^{T}\left|p(t)-p_{r, s}(t)\right| d t$ (with $T=\tau_{r}+\tau_{s}$ ) are sufficiently small (see [38, Theorem 1.3] for the details).

We describe now the geometry associated to the phase-portrait of (4.3) in order to show how to apply Theorem 3.1. We remark that similar results can be obtained for equation (4.2) with $g$ a monotone nondecreasing function bounded from below and such that $g^{\prime}(+\infty)=$ $k>0$. 
With reference to (4.3), first of all, we observe that the Poincaré map $\phi$ associated to the equivalent planar system can be decomposed as

$$
\phi=\phi_{s} \circ \phi_{r}
$$

where $\phi_{r}$ and $\phi_{s}$ are the Poincaré maps

$$
\phi_{r}: z_{0} \mapsto \zeta_{r}\left(\tau_{r}, z_{0}\right), \quad \phi_{s}: z_{0} \mapsto \zeta_{s}\left(\tau_{s}, z_{0}\right)
$$

along the time intervals $\left[0, \tau_{r}\right]$ and $\left[0, \tau_{s}\right]$ associated to the dynamical systems defined by

$$
\left\{\begin{array}{l}
\dot{x}=y \\
\dot{y}=-k x^{+}+r
\end{array}\right.
$$

and

$\left(E_{s}\right)$

$$
\left\{\begin{array}{l}
\dot{x}=y \\
\dot{y}=-k x^{+}-s .
\end{array}\right.
$$

Here $\zeta_{r}\left(\cdot, z_{0}\right)$ denotes the solution $(x(\cdot), y(\cdot))$ of $\left(E_{r}\right)$ with $(x(0), y(0))=$ $z_{0}$. Clearly, $\zeta_{s}\left(\cdot, z_{0}\right)$ is defined in the same manner with respect to system $\left(E_{s}\right)$.
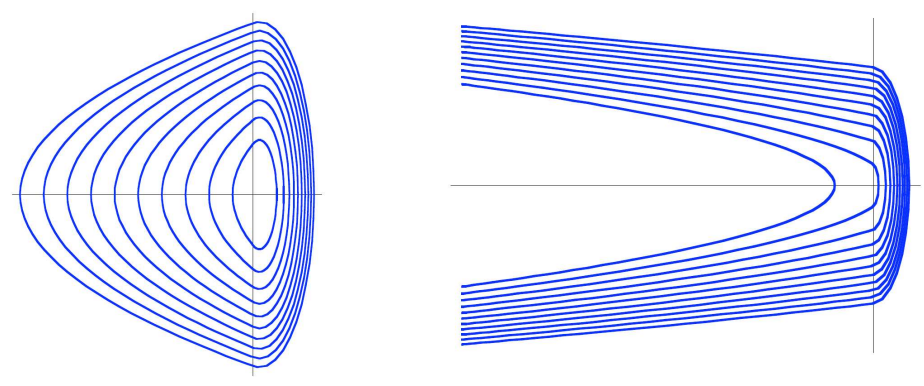

FiguRE 10. Trajectories of system $\left(E_{r}\right)$ (left) and system $\left(E_{s}\right)$ (right). For this example we have chosen $k=10, r=4$ and $s=0.5$.

The orbits of system $\left(E_{r}\right)$ correspond to the level lines of the energy

$$
\mathcal{E}_{r}(x, y):=\frac{1}{2} y^{2}+\frac{k}{2}\left(x^{+}\right)^{2}-r x .
$$

Except for the equilibrium point $(\bar{x}, 0)$ (with $\bar{x}:=\frac{r}{k}$ ), they are closed curves which are run in the clockwise sense. Moreover, their fundamental period is a monotonically nondecreasing function with respect to the energy and it tends to infinity as the energy tends to infinity.

EJQTDE, Proc. 8th Coll. QTDE, 2008 No. 14, p. 25 
On the other hand, the orbits of system $\left(E_{s}\right)$ correspond to the level lines of the energy

$$
\mathcal{E}_{s}(x, y):=\frac{1}{2} y^{2}+\frac{k}{2}\left(x^{+}\right)^{2}+s x .
$$

They are run with $y^{\prime}<0$ (see Fig. 10 and Fig. 11).

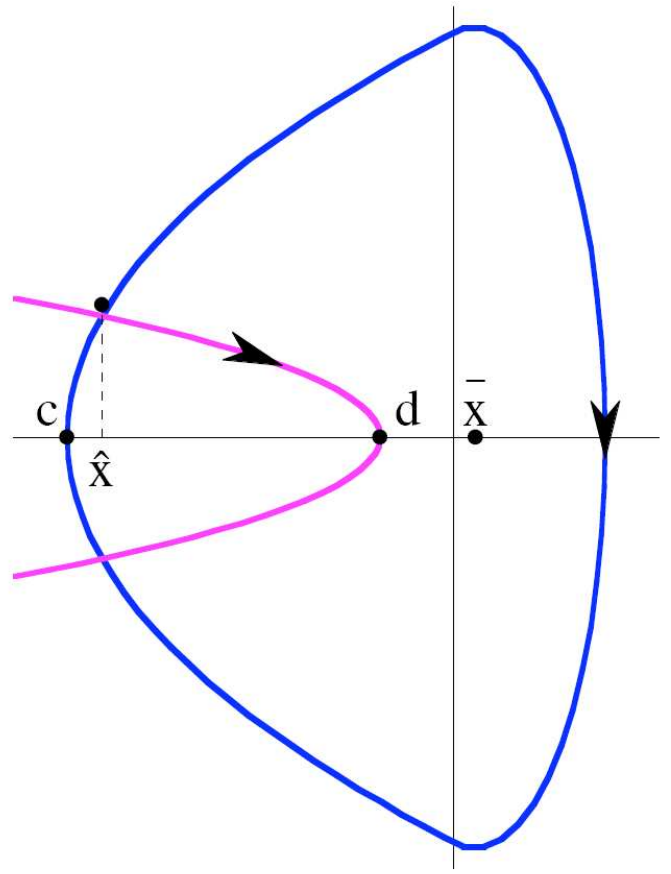

FiguRE 11. Example of a trajectory of $\left(E_{r}\right)$ (drawn with a darker line) overlapped with a trajectory of $\left(E_{s}\right)$. The times for moving a point along an orbit from $c$ to $\hat{x}$ or from $\hat{x}$ to $d$ can be explicitly determined by means of time-mappings integrals.

Since our trajectories will switch from system $\left(E_{r}\right)$ to system $\left(E_{s}\right)$ and viceversa, in order to study the effect of the forcing term $p_{r, s}(t)$, we are led to overlap the energy level lines of the two systems, as shown in Fig. 11. Next, intersecting two level lines of $\left(E_{r}\right)$ with two level lines of $\left(E_{s}\right)$, we can determine two regions, like in Fig. 12.

EJQTDE, Proc. 8th Coll. QTDE, 2008 No. 14, p. 26 


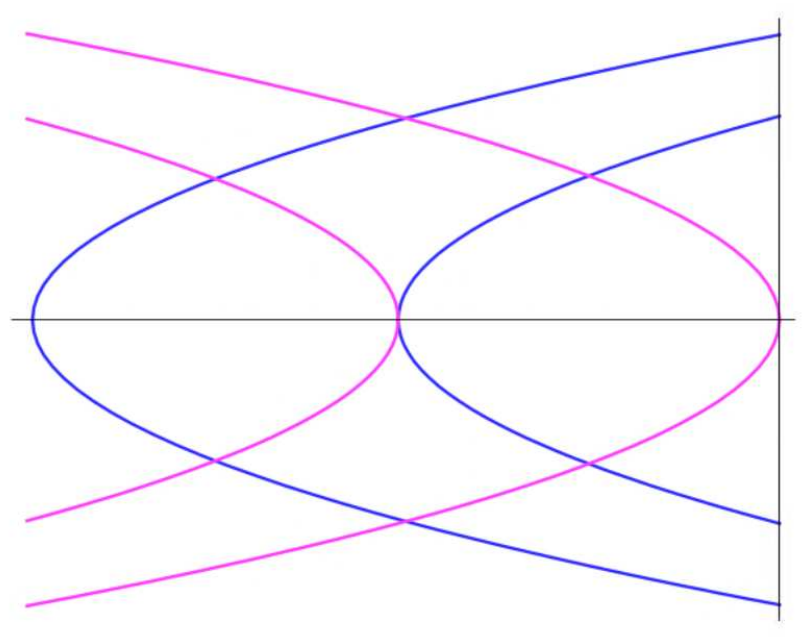

FiguRE 12. Level lines of system $\left(E_{r}\right)$ overlapped with the level lines of system $\left(E_{s}\right)$ in the left half plane $x \leq 0$. The sets $\mathcal{P}$ and $\mathcal{O}$ are those bounded by the four different level lines and contained in the lower half plane $y \leq 0$ and in the upper half plane $y \geq 0$, respectively.

In order to get periodic and chaotic solutions for the system

$$
\left\{\begin{array}{l}
\dot{x}=y \\
\dot{y}=-k x^{+}+p_{r, s}(t)
\end{array}\right.
$$

and thus for equation (4.3), we employ the following elementary procedure: we construct a set $\mathcal{P}$ in the third quadrant bounded by two level lines of $\left(E_{r}\right)$ and two level lines of $\left(E_{s}\right)$. Using the fact that the period $\mathcal{T}(\cdot)$ of the closed orbits of system $\left(E_{r}\right)$ is a strictly monotone increasing function on $[0,+\infty)$, we define the two components of $\mathcal{P}^{-}$ (here denoted by $\mathcal{P}_{1}^{-}$and $\mathcal{P}_{2}^{-2}$ ) as the intersections of $\mathcal{P}$ with the level lines of $\mathcal{E}_{r}$, so that points on the inner boundary move faster than points of the outer boundary. Hence, if we take a path $\gamma$ contained in the third quadrant and joining such two boundary segments, we'll find that (after a sufficiently long time $\tau_{r}$ ) its image through $\phi_{r}$ turns out to be a spiral-like line crossing the second quadrant at least twice. In particular, we can select two sub-paths (say $\sigma_{1}$ and $\sigma_{2}$ ) of $\gamma$ whose

\footnotetext{
2 in order to avoid confusion with the letters $r$ and $s$ already employed in equation (4.3).
}

EJQTDE, Proc. 8th Coll. QTDE, 2008 No. 14, p. 27 
images through $\phi_{r}$ cross the second quadrant. We also define a rectangular subset $\mathcal{O}$ in the second quadrant (which is just the reflection of $\mathcal{P}$ with respect to the $x$-axis) having as components of $\mathcal{O}^{-}$(denoted now by $\mathcal{O}_{1}^{-}$and $\mathcal{O}_{2}^{-}$) the intersections of $\mathcal{O}$ with two level lines of $\mathcal{E}_{s}$ and such that $\phi_{r}\left(\sigma_{1}\right)$ and $\phi_{r}\left(\sigma_{2}\right)$ admit sub-paths contained in $\mathcal{O}$ and joining $\mathcal{O}_{1}^{-}$to $\mathcal{O}_{2}^{-}$. When we switch to system $\left(E_{s}\right)$, we can prove that the images of such sub-paths through $\phi_{s}$ cross the set $\mathcal{P}$ in the right manner. Then we can apply Theorem 3.1 provided we are free to tune the switching times $\tau_{r}$ and $\tau_{s}$. The technical details for this argument can be found in [38].

Similar conclusions can be drawn for the second order ODE

$$
x^{\prime \prime}+q(t) g(x)=0,
$$

with $g$ a nonlinear term and $q(\cdot)$ a periodic step function (or a suitable small perturbation of a step function). In the case of a general (but sign changing) weight function $q(t)$, previous results for this equation have been obtained by Butler [7], Terracini and Verzini [52], Papini and Zanolin $[32,33,35]$. The detection of chaotic-like solutions for (4.6) with a positive $q(t)$ and under general conditions on $g(x)$ appears as an interesting problem which has not yet been fully investigated. Due to space limitations, we confine such study to a future work.

\section{Conclusions}

In the present article we have shown how to apply the abstract topological theory exposed in Section 2 and already developed in [34, 35] to prove the existence of chaotic dynamics for some concrete equations, whose phase-portrait recalls the geometry of the linked twist maps. Further developments in this direction can be obtained by studying new ODE models, whose Poincaré map presents features similar, but not corresponding exactly, to that of the classical LTMs. For example, frameworks like the one in Fig. 7 can be considered as well. Suitable variants of such situations will be analyzed in a future paper.

The examples of ODEs we have presented suggest the possibility of proving in an elementary but rigorous manner the presence of chaotic dynamics for a broad class of second order nonlinear ODEs with periodic coefficients (which are piecewise constant or, anyway, not far from the piecewise constant case), provided that we have the freedom to tune some switching time parameters within a certain range depending on the coefficients governing the equations. Our arguments in the proofs EJQTDE, Proc. 8th Coll. QTDE, 2008 No. 14, p. 28 
indicate that the same is true also for more general time-dependent coefficients. However, a rigorous proof in such a case would require a more delicate analysis or possibly the aid of computer assistance (as in $[3,4,26,27,57])$. This is far beyond the aims of the present paper where, instead, we wanted only to put in evidence the presence of some simple geometric configurations which appear in a natural way in various planar systems and lead to "chaos".

\section{REFERENCES}

[1] R.L. Adler, A.G. Konheim And M.H. McAndrew, Topological entropy, Trans. Amer. Math. Soc. 114 (1965), 309-319.

[2] J.C. Alexander, A primer on connectivity, Fixed point theory (Sherbrooke, Que., 1980), Lecture Notes in Math., Springer, Berlin 886 (1981), 455-483.

[3] B. BÁnhelyi, T. Csendes and B.M. Garay, Optimization and the Miranda approach in detecting horseshoe-type chaos by computer, Int. J. Bifurcation and Chaos 17 (2007), 735-747.

[4] B. Bánhelyi, T. Csendes, B.M. Garay and L. Hatvani, A computerassisted proof for $\Sigma_{3}$-chaos in the forced damped pendulum equation, (to appear).

[5] K. Burns And H. Weiss, A geometric criterion for positive topological entropy, Comm. Math. Phys. 172 (1995), 95-118.

[6] R. Burton and R.W. Easton, Ergodicity of linked twist maps, Global theory of dynamical systems (Proc. Internat. Conf., Northwestern Univ., Evanston, Ill., 1979), Lecture Notes in Math., Springer, Berlin 819 (1980), 35-49.

[7] G.J. ButLer, Rapid oscillation, nonextendability, and the existence of periodic solutions to second order nonlinear ordinary differential equations, $J$. Differential Equations 22 (1976), 467-477.

[8] A. Capietto, W. Dambrosio and D. Papini, Superlinear indefinite equations on the real line and chaotic dynamics, J. Differential Equations 181 (2002), 419-438.

[9] C. Conley, An application of Ważewski's method to a non-linear boundary value problem which arises in population genetics, J. Math. Biol. 2 (1975), 241-249.

[10] R.L. Devaney, Subshifts of finite type in linked twist mappings, Proc. Amer. Math. Soc. 71 (1978), 334-338.

[11] R.W. Easton, Isolating blocks and symbolic dynamics, J. Differential Equations 17 (1975), 96-118.

[12] D. Gale, The game of Hex and the Brouwer fixed-point theorem, Amer. Math. Monthly 86 (1979), 818-827.

[13] Z. Galias and P. Zgliczyński, Abundance of homoclinic and heteroclinic orbits and rigorous bounds for the topological entropy for the Hénon map, Nonlinearity 14 (2001), 909-932.

[14] J. Kennedy, S. KoÇaK and Y.A. Yorke, A chaos lemma, Amer. Math. Monthly 108 (2001), 411-423.

EJQTDE, Proc. 8th Coll. QTDE, 2008 No. 14, p. 29 
[15] J. Kennedy And J.A. Yorke, The topology of stirred fluids, Topology Appl. 80 (1997), 201-238.

[16] J. Kennedy And J.A. Yorke, Dynamical system topology preserved in presence of noise, Tr. J. of Mathematics 22 (1998), 379-413.

[17] J. Kennedy and J.A. Yorke, Topological horseshoes, Trans. Amer. Math. Soc. 353 (2001), 2513-2530.

[18] U. Kirchgraber And D. Stoffer, On the definition of chaos, Z. Angew. Math. Mech. 69 (1989), 175-185.

[19] W. Kulpa, The Poincaré-Miranda theorem, Amer. Math. Monthly 104 (1997), 545-550.

[20] W. Kulpa, M. Pordzik, L. Socha and M. Turzanski, $L_{1}$ cheapest paths in "Fjord scenery", European J. Oper. Res. 161 (2005), 736-753.

[21] A.C. LAzer And P.J. MCKenna, Large scale oscillatory behaviour in loaded asymmetric systems, Ann. Inst. Henry Poincaré, Analyse non lineaire 4 (1987), 244-274.

[22] A.C. LAzer And P.J. MCKenna, Large-amplitude periodic oscillations in suspension bridges: some new connections with nonlinear analysis, SIAM Review 32 (1990), 537-578.

[23] J. MAwhin, Poincaré early use of Analysis situs in nonlinear differential equations: Variations around the theme of Kronecker's integral, Philosophia Scietiae 4 (2000), 103-143.

[24] J. Mawhin, Resonance and nonlinearity: a survey. Preprint 2006.

[25] C. Miranda, Un'osservazione su un teorema di Brouwer, Boll. Un. Mat. Ital. (2) 3 (1940), 5-7.

[26] K. Mischaikow And M. MrozeK, Chaos in the Lorenz equations: a computer-assisted proof, Bull. Amer. Math. Soc. 32 (1995), 66-72.

[27] K. Mischaikow And M. Mrozek, Isolating neighborhoods and chaos, Japan J. Indust. Appl. Math. 12 (1995), 205-236.

[28] J. Moser, Stable and Random Motions in Dynamical Systems. With special emphasis on celestial mechanics, Hermann Weyl Lectures, the Institute for Advanced Study, Princeton, N. J. Annals of Mathematics Studies, No. 77. Princeton University Press, Princeton, N. J., 1973.

[29] M. Mrozek And K. WóJcik, Discrete version of a geometric method for detecting chaotic dynamics, Topology Appl. 152 (2005), 70-82.

[30] J.S. Muldowney AND D. WiLletT, An elementary proof of the existence of solutions to second order nonlinear boundary value problems, SIAM J. Math. Anal 5 (1974), 701-707.

[31] D. PAPINI, Prescribing the nodal behaviour of periodic solutions of a superlinear equation with indefinite weight, Atti Sem. Mat. Fis. Univ. Modena 51 (2003), 43-63.

[32] D. PAPini And F. ZAnolin, A topological approach to superlinear indefinite boundary value problems, Topol. Methods Nonlinear Anal. 15 (2000), 203-233.

[33] D. PAPIni And F. Zanolin, Periodic points and chaotic-like dynamics of planar maps associated to nonlinear Hill's equations with indefinite weight, Georgian Mathematical J. 9 (2002), 339-366.

EJQTDE, Proc. 8th Coll. QTDE, 2008 No. 14, p. 30 
[34] D. Papini And F. Zanolin, On the periodic boundary value problem and chaotic-like dynamics for nonlinear Hill's equations, Adv. Nonlinear Stud. 4 (2004), 71-91.

[35] D. Papini And F. Zanolin, Fixed points, periodic points, and coin-tossing sequences for mappings defined on two-dimensional cells, Fixed Point Theory Appl. 2004 (2004), 113-134.

[36] D. PAPIni And F. Zanolin, Some results on periodic points and chaotic dynamics arising from the study of the nonlinear Hill equations, Rend. Sem. Mat. Univ. Pol. Torino 65 (2007), 115-157 (Special Issue: Subalpine Rhapsody in Dynamics).

[37] A. Pascoletti, Punti fissi per mappe del piano con applicazioni alle equazioni differenziali, Tesi di laurea magistrale, University of Udine, 2007.

[38] A. Pascoletti And F. Zanolin, Example of a suspension bridge ODE model exhibiting chaotic dynamics: a topological approach, J. Math. Anal. Appl. 339 (2008), 1179-1198.

[39] M. Pireddu And F. Zanolin, Fixed points for dissipative-repulsive systems and topological dynamics of mappings defined on $N$-dimensional cells, $A d v$. Nonlinear Stud. 5 (2005), 411-440.

[40] M. Pireddu And F. Zanolin, Cutting surfaces and applications to periodic points and chaotic-like dynamics, Topol. Methods Nonlinear Anal. (to appear).

[41] H. Poincaré, Sur certaines solutions particulières du problème des trois corps, C.R. Acad. Sci. Paris 97 (1883), 251-252.

[42] H. Poincaré, Sur certaines solutions particulières du problème des trois corps, Bulletin Astronomique 91 (1884), 65-74.

[43] F. PrZytycki, Ergodicity of toral linked twist mappings, Ann. Sci. École Norm. Sup. (4) 16 (1983), 345-354.

[44] F. Przytycki, Periodic points of linked twist mappings, Studia Math. 83 (1986), 1-18.

[45] C. Rebelo and F. Zanolin, On the existence and multiplicity of branches of nodal solutions for a class of parameter-dependent Sturm-Liouville problems via the shooting map, Differential Integral Equations 13 (2000), 1473-1502.

[46] D.E. SAnderson, Advanced plane topology from an elementary standpoint, Math. Mag. 53 (1980), 81-89.

[47] S. Smale, Finding a horseshoe on the beaches of Rio, Math. Intelligencer 20 (1998), 39-44.

[48] R. SRzEDNicki, A generalization of the Lefschetz fixed point theorem and detection of chaos, Proc. Amer. Math. Soc. 128 (2000), 1231-1239.

[49] R. SRzednicki And K. Wójcik, A geometric method for detecting chaotic dynamics, J. Differential Equations 135 (1997), 66-82.

[50] R. Sturman, The linked twist map approach to fluid mixing, Dynamical Systems and Statistical Mechanics, London Mathematical Society Durham Symposium 2006. Available online at: http://www.maths.dur.ac.uk/events/Meetings/LMS/2006/DSSM/talks.html

[51] A. Szymczak, The Conley index and symbolic dynamics, Topology 35 (1996), $287-299$

EJQTDE, Proc. 8th Coll. QTDE, 2008 No. 14, p. 31 
[52] S. Terracini and G. Verzini, Oscillating solutions to second- order ODEs with indefinite superlinear nonlinearities, Nonlinearity 13 (2000), 1501-1514.

[53] P. Walters, An introduction to ergodic theory, Graduate Texts in Mathematics, Vol. 79, Springer-Verlag, New York, 1982.

[54] S. Wiggins, Chaos in the dynamics generated by sequence of maps, with application to chaotic advection in flows with aperiodic time dependence, $Z$. angew. Math. Phys. 50 (1999), 585-616.

[55] S. Wiggins and J.M. Ottino, Foundations of chaotic mixing, Philos. Trans. R. Soc. Lond. Ser. A Math. Phys. Eng. Sci. 362 (2004), 937-970.

[56] P. ZGLiczyŃski, Fixed point index for iterations of maps, topological horseshoe and chaos, Topol. Methods Nonlinear Anal. 8 (1996), 169-177.

[57] P. ZGLiCZYŃski, Computer assisted proof of chaos in the Rössler equations and in the Hénon map, Nonlinearity 10 (1997), 243-252.

[58] P. ZGLICZYŃSKI, Sharkovskii's theorem for multidimensional perturbations of one-dimensional maps, Ergodic Theory Dynam. Systems 19 (1999), 1655-1684.

[59] P. ZGLICZYŃSKI, On periodic points for systems of weakly coupled 1-dim maps, Nonlinear Anal. Ser. A: Theory Methods 46 (2001), 1039-1062.

[60] P. Zgliczyński and M. Gidea, Covering relations for multidimensional dynamical systems, J. Differential Equations 202 (2004), 32-58.

(Received August 30, 2007)

University of Udine,

Department of Mathematics and Computer Science,

Via delle Scienze 206, 33100 Udine, Italy

mailto: anna.pascoletti@dimi.uniud.it

mailto: marina.pireddu@dimi.uniud.it

mailto: fabio.zanolin@dimi.uniud.it

EJQTDE, Proc. 8th Coll. QTDE, 2008 No. 14, p. 32 